Eastern Economic Journal, 2010, 36, (284)

(C) 2010 EEA 0094-5056/10

www.palgrave-journals.com/eej/

\title{
List of Reviewers 2009
}

\author{
Eastern Economic Journal (2010) 36, 284. doi:10.1057/eej.2010.13
}

The Editors and Editorial Board wish to acknowledge the assistance of the following colleagues who reviewed articles during 2009 for their contributions of time and experience. Without their dedication, our work in publishing this Journal could not go forward.

\begin{tabular}{|c|c|c|c|}
\hline Monika AbuGhazaleh & Karin Margret Edmark & Christopher C. Klein & Subramaniam \\
\hline Andrea Ajello & Tore Ellingsen & Steven Klepper & Ramanarayanan \\
\hline Jesper Andersen & Craig Ellis & Kristin Klopfenstein & Miguel Ramirez \\
\hline Kathleen Arano & Tisha Lin Nakao Emerson & Kim Klyver & William Rand \\
\hline Muhammad Asali & Molly Espey & S. F. Koch & Bhaskara Rao \\
\hline Carlos J. Asarta & Amy Farmer & Jonathan T. Kolstad & Randall Reback \\
\hline Alan Barrett & Helga Fehr-Duda & Peter Krause & Daniel I. Rees \\
\hline Siegfried Berninghaus & Ida Ferrara & Tomi Kyyra & Cordelia Reimers \\
\hline Robert Bifulco & Kenneth Fjell & Johann Lambsdorff & Elke Renner \\
\hline Roberto M. Billi & Jason Fletcher & Gauthier Lanot & John Robst \\
\hline Christian Bjørnskov & Kevin F. Forbes & William D. Lastrapes & Armando E. Rodriguez \\
\hline Hans Bonesrønning & Nicolai Foss & Blake LeBaron & Brian Roe \\
\hline William Bosshardt & Kosei Fukuda & Michael A. Leeds & Christian Roessler \\
\hline Pablo Branas-Garza & Marcos Gallacher & Andrew Leigh & Joshua L. Rosenbloom \\
\hline Massimiliano Bratti & Lisa Giddings & Yue Li & Don Ross \\
\hline Paul Brenton & Andrew Gill & Marko Lindroos & Stephen L. Ross \\
\hline Meta Brown & John H. Goddeeris & Charles Link & Marina V. Rosser \\
\hline Thomas Brown & Stephan Gohmann & Kim Hiang Liow & Gregoire Rota Graziosi \\
\hline Mark Burkey & Orlando Gomes & John A. List & David B. Ryden \\
\hline Barbara Burnell & Umberto Gostoli & Colin Martyn Lizieri & Rexford E. Santerre \\
\hline Marco Cagetti & Mary Graham & Youenn Lohéac & Malcolm Sawyer \\
\hline Dario Caldara & Paul Grootendorst & John B. Loomis & Robert Scharff \\
\hline Lindsay Calkins & G. Emmanuel Guindon & Michael Lynn & Maarten Pieter Schinkel \\
\hline Christian Calmes & Bjorn Gustafsson & Thierry Madies & Martin Bryan Schmidt \\
\hline James Cardon & Marco A. Haan & Mahoney & Pamela Schmitt \\
\hline Thomas F. Cargill & William Hamlen & E. Marcotte & Friedrich Schneider \\
\hline Steven Peter Cassou & Nobuyuki Hanaki & Joseph R. Mason & Bisakha Sen \\
\hline Ken Cavalluzzo & Denise Hare & Toshihiro Matsumura & Mark Setterfield \\
\hline Duncan Chaplin & Shaun Hargreaves Heap & Ann Mari May & Andrew Sfekas \\
\hline Nicolas Depetris Chauvin & Jonathan Haughton & Leslie McCall & Ian M. Sheldon \\
\hline Jacopo Cimadomo & Ling T. He & Catherine L. McDevitt & Molly Sherlock \\
\hline George Clarke & Andrew J. Healy & John M. McDowell & Peter Skott \\
\hline Sofronis Clerides & Joni Hersch & Leslie McGranahan & Brian W. Sloboda \\
\hline Alex Coad & Lisa Herzog & Melville McMillan & James L. Smith \\
\hline Dennis Coates & Alexander Hijzen & Monika Merz & Paul Smoke \\
\hline Simona Cociuba & Hines & Jochen Michaelis & Sara Solnick \\
\hline Ethan Cohen-Cole & Christian L. Holzner & Ted R. Miller & J. Farley Ordovensky \\
\hline Riccardo Corradini & Anthony C. Homan & V. Mollick & \\
\hline Souleymane Coulibaly & Ed Hopkins & Mark Montgomery & Omari H. Swinton \\
\hline Tom Coupé & Julius Horvath & Juan Carlos Moreno-Brid & Chor Foon Tang \\
\hline James Cover & Peter Davenport Hull & Peter Mueser & Frank Tinari \\
\hline Erik Craft & Christophe Hurlin & Matthew G. Nagler & Mark Toma \\
\hline Ana C. Dammert & Andrew Hussey & Julie A. Nelson & Maude \\
\hline Ronald B. Davies & Ryan Huston & Austin Nichols & Toussaint-Comeau \\
\hline Lewis Davis & Thomas R. Ireland & Neil Niman & Terje Vassdal \\
\hline Joseph DeJuan & Andreas Irmen & Alfonso Palacio-Vera & Jessica Vistnes \\
\hline Steven Deller & Daniel Johnson & Yongjin Park & Gary Wagner \\
\hline Jeffrey DeSimone & Robert J. Johnston & Randall Parker & Dave Weimer \\
\hline Dennis A. V. Dittrich & Stefan Jungblut & Susana Peralta & Catherine J. Weinberger \\
\hline Robert Dolan & Robert Kaestner & Mark Allan Pingle & Jeanne Wendel \\
\hline Matthew Doyle & Charlene Marie & Andrew Plantinga & Kip Womack \\
\hline Scott David Drewianka & Kalenkoski & J. Gary Polhill & Vivian Y. Wu \\
\hline Julan Du & Pavel Kapinos & Niklas Potrafke & Huan Xie \\
\hline Kevin C. Duncan & Donald S. Kenkel & Mozaffar Qizilbash & Bo Zhao \\
\hline Alex Dupuy & Rasheda Khanam & Martin Quaas & Benjamin Chris Zissimos \\
\hline Damien Echevin & Ahmed Khwaja & Kevin G. Quinn & Sarah Zubairy \\
\hline
\end{tabular}

